\title{
When is the Set of Base-Parameter Values Physically Impossible?
}

\author{
Koji Yoshida*1 Koichi Osuka*1 $^{*}$ Hirokazu Mayeda*2 $^{*}$ Toshiro Ono*1
}

\begin{abstract}
A method has been proposed to judge if a set of base-parameter values for the dynamic model of a manipulator determines the inertial matrix of the dynamic model to be positive definite for each configuration of the manipulator or not, when continuous change of each joint variable of the manipulator is approximately considered as a finite set of discrete points. If a set of base-parameter values is judged not to do, it is physically impossible. The method can be executed on computers. The method is useful to examine a set of estimated base-parameter values. A method also has been proposed to modify the estimated base-parameter values such that the set of them determines the inertial matrix to be always positive definite if it is judged not to do.
\end{abstract}

Key Words: Manipulator, Dynamic Model, Inertial Matrix, Positive Definite, Parameter Estimation

\section{Introduction}

For the model-based control of a manipulator, it is very crucial to obtain an accurate dynamic model of the manipulator motion [1]. The dynamic model of the manipulator consisting of rigid links is described as a set of nonlinear differential equations involving various constant parameters: kinematic parameters, link inertial parameters, and dynamic parameters of driving systems. Hence, accurate values of the parameters are required to obtain an accurate dynamic model. The values of the kinematic parameters are obtained from design specifications or kinematic calibration. After obtaining them, for the purpose of determining the dynamic model of the manipulator it is sufficient for us to obtain the dynamic parameters of driving systems and the values of each element of a base parameter set [2] [3] which is defined to be a minimum set of inertial parameters whose values can determine the dynamic model uniquely. The estimation methods of those parameter values have been proposed by several authors $[4] \sim[7]$, and they can be classified into two groups [8] [9].

A base parameter set generates each element of the inertial matrix of the dynamic model of a manipulator. We can then determine the inertial matrix after estimating a set of base-parameter values. Though it is the fact that the inertial matrix is positive definite for each configuration of the manipulator, it may happen that the inertial matrix is not always positive definite if it is determined by a set of estimated base-parameter values. If a set of base-parameter values de-

原稿受付 1994 年 11 月 18 日

${ }^{* 1}$ Osaka Prefecture University

${ }^{* 2}$ Ritsumeikan University termines such inertial matrix, it is physically impossible and it is needless to examine the accuracy of the values as far as we use the dynamic model derived under the assumption that all the links of the manipulator are rigid. The dynamic model that is determined by such a set of base-parameter values would express nothing in the physical world. If the manipulator were controlled by using such a set of baseparameter values, a good performance of the manipulator would not be ensured, and if the manipulator motion were simulated, the results would not be worth believing. Hence, it is worth while to make it clear which set of base-parameter values is physically possible.

There has not been any study about the relationship between a set of base-parameter values and the physical possibility or impossibility of the dynamic model. Hence, as the first step in the context of making clear which set of baseparameter values is physically possible, in this paper we will propose a method to judge if a set of base-parameter values determines the inertial matrix to be positive definite for each configuration of the manipulator or not, when we approximately consider the continuous change of each joint variable of the manipulator as a finite set of discrete points. The method can be executed on computers. Using this method we can judge if a set of estimated base-parameter values is "possible" or not. Here we use "possible" in the sense that the set of base-parameter values determines the inertial matrix to be positive definite for each configuration of the manipulator. If a set of base-parameter values is not "possible", it is physically impossible. The physical impossibility of a set of estimated base-parameter values is caused by the biased base-parameter values of the set. We actually estimate a set of base-parameter values in the presence of 
a noise on data, modeling error, and so on, hence, we are forced to have biased base-parameter values more or less. Then, no estimation method would ensure to give a set of estimated base-parameter values to be always "possible". If we unfortunately estimate a set of base-parameter values not to be "possible", should we try to estimate again without any strategy? Otherwise should we try to develop the ultimate estimation method that gives always "possible" set of base-parameter values? Both would be just laborious and fruitless. Hence we will also propose one method to modify the estimated base-parameter values for the set of them to be at least "possible" if we judge it is not. In the modification we will make the best of the originally estimated baseparameter values. The modification of the estimated baseparameter values might reduce the accuracy of some values, however, it would be better that a set of base-parameter values that we use is "possible" than that it is not "possible", i.e. physically impossible.

\section{Inertial Matrix}

The inertial matrix of the dynamic model of a manipulator will be explained briefly in this chapter.

In this paper, we consider the manipulator having openloop kinematic chain mechanisms with $\mathrm{N}$ links and revolute joints only. We number the links successively from 0 to $\mathrm{N}$, link 0 is a stationary pillar. We then apply the method in [2], [3] to attach a coordinate system $\left(o_{i} ; \boldsymbol{x}_{i}, \boldsymbol{y}_{i}, \boldsymbol{z}_{i}\right)$ to link $i$. $o_{i}$ denotes the origin of the coordinate system. Let $\theta_{i}$ denote a joint variable. Then, $3 \times 3$ rotation matrices are defined. Let $m_{i}$ and $\boldsymbol{I}_{i}$ be the mass and the inertia tensor around $o_{i}$ of link $i$, respectively. Let $\boldsymbol{r}_{i}$ and $\boldsymbol{l}_{i}$ be the vectors from $o_{i}$ to the center of mass of link $i$ and $o_{i+1}$, respectively. The representation of any vector $\boldsymbol{v}$ or any tensor $\boldsymbol{T}$ about $\left(o_{i} ; \boldsymbol{x}_{i}, \boldsymbol{y}_{i}, \boldsymbol{z}_{i}\right)$ are denoted by ${ }^{i} \boldsymbol{v}$, or ${ }^{i} \boldsymbol{T}$, respectively. Then, ${ }^{i} \boldsymbol{l}_{i},{ }^{i} \boldsymbol{r}_{i}$, and ${ }^{i} \boldsymbol{I}_{i}$ are constant vectors and a constant matrix. For each link $i$ we have ten parameters: $m_{i}$, three elements of $m_{i}{ }^{i} \boldsymbol{r}_{i}$, and six independent elements of ${ }^{i} \boldsymbol{I}_{i}$, each of these ten parameters will be called link inertial parameter. Any linear combination of the link inertial parameters is defined to be an inertial parameter [2].

Then we introduce the following notation:

$$
\begin{gathered}
\boldsymbol{M}_{i}=\sum_{j=i}^{N} m_{j}, \\
\boldsymbol{R}_{i}=\boldsymbol{M}_{i+1} \boldsymbol{l}_{i}+m_{i} \boldsymbol{r}_{i}, \\
\boldsymbol{S}_{i}=\sum_{j=i}^{N} \boldsymbol{R}_{j}, \\
\boldsymbol{J}_{i}=\boldsymbol{I}_{i}+\boldsymbol{M}_{i+1}\left[\left(\boldsymbol{l}_{i} \cdot \boldsymbol{l}_{i}\right) \boldsymbol{E}-\boldsymbol{l}_{i} \otimes \boldsymbol{l}_{i}\right]
\end{gathered}
$$

$$
\boldsymbol{L}_{j, i}=\sum_{s=j}^{i-1} l_{s}
$$

where $\boldsymbol{E}$ is unit tensor of rank 2. Symbols $\cdot, \times$, and $\otimes$ denote the operations of inner product, cross product, and tensor product for vectors, respectively. $\boldsymbol{M}_{i}, \boldsymbol{R}_{i}$, and $\boldsymbol{J}_{i}$ are the moments of order 0,1 , and 2 , respectively, for the augmented link [10] of link $i$ around $o_{i}$. Then ${ }^{i} \boldsymbol{R}_{i}$ and ${ }^{i} \boldsymbol{J}_{i}$ are constant vector and constant matrix. Note that $\boldsymbol{M}_{i}$ and all entries of ${ }^{i} \boldsymbol{R}_{i}$ and ${ }^{i} \boldsymbol{J}_{i}$ are inertial parameters.

In general, the dynamic model of the manipulator can be written as

$$
\boldsymbol{\tau}=\boldsymbol{H}(\boldsymbol{\theta}) \ddot{\boldsymbol{\theta}}+\boldsymbol{B}(\boldsymbol{\theta}, \dot{\boldsymbol{\theta}}) \dot{\boldsymbol{\theta}}+\boldsymbol{G}(\boldsymbol{\theta})+\boldsymbol{V} \dot{\boldsymbol{\theta}}+\boldsymbol{C} \operatorname{sgn}(\dot{\boldsymbol{\theta}})
$$

where $\boldsymbol{\tau}=\left[\begin{array}{llll}\tau_{1} & \cdots & \tau_{N}\end{array}\right]^{t}$ is the joint torque vector and $\boldsymbol{\theta}=\left[\begin{array}{llll}\theta_{1} & \cdots & \theta_{N}\end{array}\right]^{t}$ is the joint variable vector. The superscript $t$ indicates transposition. $\boldsymbol{H}(\boldsymbol{\theta})$ is $\mathrm{N} \times \mathrm{N}$ inertial term matrix, $\boldsymbol{B}(\boldsymbol{\theta}, \dot{\boldsymbol{\theta}})$ is the Coriolis and centrifugal force term matrix, and $\boldsymbol{G}(\boldsymbol{\theta})$ is N-dimensional gravity term vector. $\boldsymbol{V} \dot{\boldsymbol{\theta}}$ represents viscous friction force and $\boldsymbol{C} \operatorname{sgn}(\dot{\boldsymbol{\theta}})$ Coulomb dry friction force where $\boldsymbol{V}$ and $\boldsymbol{C}$ are positive definite diagonal matrices, and $\operatorname{sgn}(\dot{\boldsymbol{\theta}})$ is the column vector of $\operatorname{sgn}\left(\dot{\theta}_{i}\right)(i=1, \ldots, N)$. The $(i, j)$-th element of inertial term matrix $\boldsymbol{H}(\boldsymbol{\theta})$ will be denoted by $H(i, j)$. Then it is described as [2]

$$
\begin{aligned}
H(i, j)= & \boldsymbol{z}_{i} \cdot\left(\sum_{s=i}^{N} \boldsymbol{J}_{s}\right) \boldsymbol{z}_{j} \\
& +\boldsymbol{z}_{i} \cdot\left\{\sum _ { s = i } ^ { N - 1 } \left[2\left(\boldsymbol{l}_{s} \cdot \boldsymbol{S} \boldsymbol{R}_{s+1}\right) \boldsymbol{E}\right.\right. \\
& \left.\left.-\boldsymbol{l}_{s} \otimes \boldsymbol{S} \boldsymbol{R}_{s+1}-\boldsymbol{S} \boldsymbol{R}_{s+1} \otimes \boldsymbol{l}_{s}\right]\right\} \boldsymbol{z}_{j} \\
& +\boldsymbol{z}_{i} \cdot\left[\left(\boldsymbol{L}_{j, i} \cdot \boldsymbol{S} \boldsymbol{R}_{i}\right) \boldsymbol{E}-\boldsymbol{L}_{j, i} \otimes \boldsymbol{S} \boldsymbol{R}_{i}\right] \boldsymbol{z}_{j}
\end{aligned}
$$

for $1 \leq j \leq i \leq N$, and $H(i, j)=H(j, i)$ for $i<j$.

Using the rotation matrices and ${ }^{i} \boldsymbol{z}_{i}=\left[\begin{array}{lll}0 & 0 & 1\end{array}\right]^{t}$, we evaluate each $H(i, j)$ about an appropriate coordinate system, then it is described in the following form:

$$
\sum_{v=1}^{T_{b}} p_{b_{v}} f_{b_{v}}(\boldsymbol{\theta})
$$

where $f_{b_{v}}(\boldsymbol{\theta})$ is a polynomial of trigonometric functions of $\theta_{i}$ for $1 \leq i \leq N$. ( $f_{b_{v}}$ is allowed to be a constant function), and $p_{b_{v}}$ is a base parameter.

\section{A Condition for a Set of Base-Parameter Values to be "possible"}

In this chapter, first, a necessary and sufficient condition for the inertial matrix to be positive definite will be derived. 
As shown below, the condition is described as a system of $\mathrm{N}$ inequalities, each of the $N$ inequalities can be expressed as

$$
\sum_{j=1}^{n_{i}} p_{j} f_{j}(\boldsymbol{\theta})>0
$$

where $p_{j}$ is a parameter generated by the base parameter set and $f_{j}(\boldsymbol{\theta})$ is a polynomial of trigonometric functions of $\theta_{i}$ for $1 \leq i \leq N$. Each inequality holds for each configuration of the manipulator. Conversely, based on this fact and assuming all $p_{j}$ as unknowns, we can obtain the domain of the solutions of $n_{i}$-tuples: $\left(p_{1}, \ldots, p_{n_{i}}\right)$ for the inequality to hold for each $\theta_{i}$ and for each $1 \leq i \leq N$. Then a set of base-parameter values is "possible" if and only if the $n_{i}$ tuples that is determined by the set of base-parameter values is in the domain. The goal of this chapter is to obtain the domain.

\subsection{A Condition for the Inertial Matrix to be Positive Definite}

In this section, we will derive a necessary and sufficient condition for the inertial matrix to be positive definite using the elements of the inertial matrix. Taking advantage that the inertial matrix is symmetric, we apply Sylvester's theorem to the inertial matrix. Then, the inertial matrix $\boldsymbol{H}(\boldsymbol{\theta})$ is positive definite if and only if all the leading principal minors of it are positive. We have hence a system of $N$ inequalities because we consider the $N$ degree-of-freedom manipulator. Since each element of $\boldsymbol{H}(\boldsymbol{\theta})$ is described as shown in the preceding chapter and leading principal minors are calculated by only multiplication of elements and addition and/or subtraction of the products, each of the $N$ inequalities can be described as

$$
\sum_{v=1}^{T} p_{v} f_{v}(\boldsymbol{\theta})>0
$$

where $p_{v}$ is a parameter generated by base parameter set and $f_{v}(\boldsymbol{\theta})$ is a polynomial of trigonometric functions of $\theta_{i}$ for $1 \leq i \leq N$. $T$ is the number of the terms of a leading principal minor that is considered.

We remark here 3 items. First, after calculating a principal minor, we can delete the linear dependency among $f_{1}, \ldots, f_{n}$ and $p_{1}, \ldots, p_{n}$, thus we can modify (10) as

$$
\sum_{v=1}^{T_{m}} p_{m_{v}} f_{m_{v}}(\boldsymbol{\theta})>0
$$

where $p_{m_{v}}$ is a parameter generated by base parameter set and $f_{m_{1}}(\boldsymbol{\theta}), \ldots, f_{m_{T_{m}}}(\boldsymbol{\theta})$ are linearly independent. Secondly, $p_{m_{v}}\left(v=1, \ldots, T_{m}\right)$ is a parameter that is a sum of products of some base parameters, for example, when the principal minor is 2 nd order, $p_{m_{v}}$ may be described like $p_{m_{v}}=\left(p_{b_{1}}\right)^{2}-p_{b_{1}} p_{b_{2}}+p_{b_{3}} p_{b_{4}}$ where $p_{b_{1}}, p_{b_{2}}, p_{b_{3}}$, and $p_{b_{4}}$ are base parameters. Hence, $p_{m_{v}}$ is continuous on the continuous change of base parameters. Finally, any $f_{m_{v}}(\boldsymbol{\theta})$ is a continuous and bounded function because it is a polynomial of trigonometric functions.

Consequently, we obtain the following system of $N$ inequalities as a necessary and sufficient condition for inertial matrix to be positive definite.

$$
\begin{gathered}
\sum_{v_{1}=1}^{n_{1}} p_{m 1_{v_{1}}} f_{m 1_{v_{1}}}(\boldsymbol{\theta})>0 \\
\sum_{v_{2}=1}^{n_{2}} p_{m 2_{v_{2}}} f_{m 2_{v_{2}}}(\boldsymbol{\theta})>0 \\
\ldots \\
\sum_{v_{i}=1}^{n_{i}} p_{m i_{i}} f_{m i_{v_{i}}}(\boldsymbol{\theta})>0 \\
\ldots \\
\sum_{v_{N}=1}^{n_{N}} p_{m_{N} v_{N}} f_{m_{N} v_{N}}(\boldsymbol{\theta})>0
\end{gathered}
$$

where $p_{m i v_{i}}\left(i=1, \cdots, N, \quad v_{i}=1, \cdots, n_{i}\right)$ is a parameter generated by base parameter set, and $f_{m i_{v_{i}}}(\boldsymbol{\theta})(i=$ $\left.1, \cdots, N, v_{i}=1, \cdots, n_{i}\right)$ is a polynomial of trigonometric functions of $\theta_{i}$ for $1 \leq i \leq N$, besides $f_{m i_{1}}, f_{m i_{2}}, \ldots, f_{m i_{n_{i}}}$ are linearly independent for $1 \leq i \leq N$.

The goal of this chapter is to show the subset of $\mathrm{R}^{n_{i}}$ of which the $n_{i}$-tuples $\left(p_{m i_{1}}, \ldots, p_{m i_{n_{i}}}\right)$ must be the element when the $n_{i}$-tuples is determined by a set of base-parameter values. $\mathrm{R}^{n_{i}}$ denotes the vector space consisting of $n_{i}$-tuples. For the purpose, assuming $p_{m i_{1}}, \cdots, p_{m i_{n}}$ to be the unknowns, we will show the set of the solutions for the inequality $\sum_{v_{i}=1}^{n_{i}} p_{m i_{v_{i}}} f_{m i_{v_{i}}}(\boldsymbol{\theta})>0$ to hold for each $\theta_{i}$ and for each $1 \leq i \leq N$

\section{2 Approximation of the Inequalities}

Each of the $N$ inequalities, derived in the preceding section, holds for each $\theta_{i}$ and for each $1 \leq i \leq N$. Then we must investigate the inequality when the functions change continuously. However, we will approximately consider each function as a finite set of discrete points for simplicity, thereby we obtain a system of finite linear inequalities instead of the inequality containing functions. In this section we will explain the approximation.

It is sufficient for us to consider the joint variables $\theta_{i}$ as $0 \leq \theta_{i}<2 \pi$ for each $1 \leq i \leq N$, hence $2 \pi$ is divided into $m$ pieces and $\theta_{i}$ is approximately considered to take these discrete values. If $\theta_{i}$ takes $\frac{2 \pi}{m} k$ where $k$ is integer $(0 \leq k<m)$, it is denoted by $\theta_{i}[k]$. Thus,

$$
\theta_{i}[0]=0, \theta_{i}[1]=\frac{2 \pi}{m}, \cdots, \theta_{i}[m-1]=2 \pi-\frac{2 \pi}{m} .
$$

Then substituting $\theta_{1}[k 1], \theta_{2}[k 2], \theta_{3}[k 3], \cdots, \theta_{N}[k N]$ into $f_{m i v_{i}}(\boldsymbol{\theta})$, we can obtain a real value and will denote it by 
$f_{m i}(k 1, k 2, \cdots, k N)$. Thus we obtain the system of finite linear inequalities:

$$
\begin{aligned}
& \left.\sum_{v_{i}=1}^{n_{i}} p_{m i v_{i}} f_{m i v_{i}}^{(0,} \quad 0, \cdots, 0\right)>0 \\
& \left.\sum_{v_{i}=1}^{n_{i}} p_{m i v_{i}} f_{m i v_{i}}^{(0,}, 0, \cdots, 1\right)>0 \\
& \left.\sum_{v_{i}=1}^{n_{i}} p_{m i v_{i}} f_{m i}{ }_{v_{i}}^{(m,}, m, \cdots, m\right)>0,
\end{aligned}
$$

approximating the inequality:

$$
\sum_{v_{i}=1}^{n_{i}} p_{m i_{i}} f_{m i_{v_{i}}}(\boldsymbol{\theta})>0
$$

If we take $m$ large enough, we can obtain good approximation of (15) for each $\theta_{i}$ and for each $1 \leq i \leq N$.

We introduce some symbols for convenience. vector $\boldsymbol{p}_{i}$ and $\boldsymbol{f}_{i}$ are defined as

$$
\begin{gathered}
\boldsymbol{p}_{i}=\left[\begin{array}{llll}
p_{m i_{1}} & p_{m i_{2}} & \cdots & p_{m i_{n_{i}}}
\end{array}\right]^{t} \\
\boldsymbol{f}_{i}=\left[\begin{array}{llll}
f_{m i_{1}}(\boldsymbol{\theta}) & f_{m i_{2}}(\boldsymbol{\theta}) & \cdots & f_{m i_{n_{i}}}(\boldsymbol{\theta})
\end{array}\right]^{t}
\end{gathered}
$$

and vector $\boldsymbol{f}_{i}^{1}$ is defined as

$$
\boldsymbol{f}_{i}^{1}=\left[f_{m i 1}{ }^{(0,0, \cdots, 0)} f_{m i_{2}}{ }^{(0,0, \cdots, 0)} \cdots f_{m i_{n_{i}}}{ }^{(0,0, \cdots, 0)}\right]^{t} .
$$

Then the first inequality in (14) can be expressed as $\boldsymbol{f}_{i}^{1} \cdot \boldsymbol{p}_{i}>$ 0 . In the same way, vector $f_{i}^{k}$ is defined to express the $\mathrm{k}$-th inequality from top in (14), and vector $\boldsymbol{f}_{i}^{q}$ is defined as

$$
\boldsymbol{f}_{i}^{q}=\left[\begin{array}{lll}
f_{m i 1}{ }^{(m, m, \cdots, m)} & \cdots & \left.f_{m i}{ }_{n_{i}}^{(m, m}, \cdots, m\right)
\end{array}\right]^{t},
$$

where $q=m^{m}$. Finally, matrix $\boldsymbol{F}_{i}$ is defined as

$$
\boldsymbol{F}_{i}=\left(\begin{array}{lllllll}
\boldsymbol{f}_{i}^{1} & \vdots & \boldsymbol{f}_{i}^{2} & \vdots & \cdots & \vdots & \boldsymbol{f}_{i}^{q}
\end{array}\right) .
$$

Then the system of finite linear inequalities (14) is described as

$$
\boldsymbol{F}_{i}^{t} \boldsymbol{p}_{i}>\mathbf{o}
$$

Each vector inequality holds for every component individually.

\subsection{Existence of Solutions}

In this section we will ensure that the system (21) has solutions.

We begin with a lemma concerning the dual systems

$$
\boldsymbol{A}^{t} \boldsymbol{p} \geq \mathbf{o} \text { and } \boldsymbol{A} \boldsymbol{x}=\mathbf{o}, \boldsymbol{x} \geq \mathbf{o}
$$

where

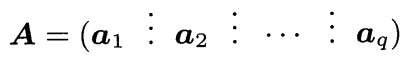

is a $q$-columned matrix with arbitrary real entries, $\boldsymbol{p}$ and $\boldsymbol{x}$ denote vectors. Each vector inequality and each vector equality holds for every component individually.

Lemma 1: The system

$$
\boldsymbol{A}^{t} \boldsymbol{p}>\mathbf{o}
$$

possesses solutions if and only if the system of equations

$$
\boldsymbol{A x}=\mathbf{o}, \boldsymbol{x} \geq \mathbf{0}
$$

possesses only a solution $\boldsymbol{x}=\mathbf{o}$.

Proof: As a corollary of Tucker's theorem [11] we can prove the lemma.

Then, we have the following theorem.

Theorem 1: The system of finite linear inequalities (21) possesses solutions.

Proof: We begin with showing that there is one constant function among

$$
f_{m i_{1}}(\boldsymbol{\theta}), \quad f_{m i_{2}}(\boldsymbol{\theta}), \quad \cdots, f_{m i_{n_{i}}}(\boldsymbol{\theta})
$$

for each $1 \leq i \leq N$.

The functions are obtained through calculating the principal minor of $\boldsymbol{H}(\boldsymbol{\theta})$. Let $\operatorname{det} \boldsymbol{H}_{r}$ denote the $r$-th order principal minor $(1 \leq r \leq N)$, then it is also described as

$$
\operatorname{det} \boldsymbol{H}_{r}=\sum_{\sigma \in S_{r}} \operatorname{sgn}(\sigma) H(1, \sigma(1)) \cdots H(r, \sigma(r))
$$

where $\sigma$ denotes a permutation of the index set $1, \ldots, r, S_{r}$ denotes the set consisting of all permutations, and $\operatorname{sgn}(\sigma)$ denotes the sign of permutation $\sigma$. Then $\operatorname{det} \boldsymbol{H}_{r}$ contains the term produced by $\prod_{s=1}^{r} H(s, s)$. On the other hand, from (7) each $(i, i)$-th entry of inertial matrix $\boldsymbol{H}(\boldsymbol{\theta})$ is described as

$$
H(i, i)=\boldsymbol{J}_{i}^{z}+G
$$

where $G$ is a function of $\theta_{s}(i+1 \leq s \leq N)$ generated by base parameters, and $\boldsymbol{J}_{i}^{z}$ is $(3,3)$-th element of ${ }^{i} \boldsymbol{J}_{i}$. Hence, $\operatorname{det} \boldsymbol{H}_{r}$ contains the term $\prod_{s=1}^{r} \boldsymbol{J}_{s}^{z}$ which is not multiplied by any function. This term will not be canceled by any other term, we will show it below. The parameter $\boldsymbol{J}_{1}^{z}$ only appears in $H(1,1)$, hence, if

$$
\operatorname{sgn}\left(\sigma^{\prime}\right) H\left(1, \sigma^{\prime}(1)\right) \cdots H\left(r, \sigma^{\prime}(r)\right) \quad\left(\sigma^{\prime} \in S_{r}\right)
$$

contains the term that cancels $\prod_{s=1}^{r} \boldsymbol{J}_{s}^{z}$, then (28) must contain $H(1,1)$. Hence, (28) must contain one of $H(2,2), H(2,3), \cdots, H(2, r)$ except $H(2,1)$, but $\boldsymbol{J}_{2}^{z}$ does not 
appear in $H(2, s)$ for $3 \leq s \leq r$, which we can see through lengthy calculation of $H(2, s)$ for $3 \leq s \leq r$. Therefore (28) must contain $H(2,2)$. By a similar argument, consequently, (28) must coincide with $\prod_{s=1}^{r} H(s, s)$, hence $\prod_{s=1}^{r} \boldsymbol{J}_{s}^{z}$ is not canceled. Then each principal minor contains the term that include $\prod_{s=1}^{r} \boldsymbol{J}_{s}^{z}$ and that is not multiplied by any function, hence we can make $f_{m i} n_{i}(\boldsymbol{\theta})$ to be constant function 1 .

Now we have $\boldsymbol{f}_{i}^{j}$ as

$$
\boldsymbol{f}_{i}^{j}=\left[\begin{array}{lll}
f_{i 1}^{j} & f_{i_{2}}^{j} \cdots f_{i(q-1)}^{j} & 1
\end{array}\right]^{t}
$$

where $f_{i}^{j}$ for $1 \leq k \leq q-1$ are real entries. Hence, we can easily show that the system

$$
\boldsymbol{F}_{i} \boldsymbol{x}=\mathbf{o}, \boldsymbol{x} \geq \mathbf{0}
$$

has only a solution $\boldsymbol{x}=\mathbf{o}$.

From Lemma 1 the system (21) has solutions.

\subsection{A Set of Solutions}

In this section we will show the set of the solutions of the system

$$
\boldsymbol{F}_{i}^{t} \boldsymbol{p}_{i}>\mathbf{o}
$$

for $i=1, \cdots, N$. It will be shown as a subset of $\mathrm{R}^{n_{i}}$.

First, we will investigate a subset of solutions of the system

$$
\boldsymbol{F}_{i}^{t} \boldsymbol{p}_{i} \geq \mathbf{0}
$$

for $i=1, \cdots, N$ and will denote it by $\mathrm{D}_{i}$. Secondly, we will investigate a subset of the solutions of the equality $\boldsymbol{f}_{i}^{k} \cdot \boldsymbol{p}_{i}=0$ for $k=1, \cdots, q$, which will be denoted by $\mathbf{S}_{i}^{k}$. Then we will get rid of the intersection of $D_{i}$ and the union of $\mathrm{S}_{i}^{1}, \mathrm{~S}_{i}^{2}, \cdots, \mathrm{S}_{i}^{q}$ from $\mathrm{D}_{i}$. We can ensure that the system (31) has solutions except $\boldsymbol{p}_{i}=\mathbf{o}$ because we have shown the existence of the solutions of the system $\boldsymbol{F}_{i}^{t} \boldsymbol{p}_{i}>\mathbf{o}$.

We introduce some terms and symbols[11]. Let $\mathrm{A}$ be a subset of $\mathrm{R}^{n_{i}}$, then we denote by $\mathrm{A}^{*}$ the set of all $\boldsymbol{y} \in \mathrm{R}^{n_{i}}$ such that $\boldsymbol{x} \cdot \boldsymbol{y} \geq 0$ for each $\boldsymbol{x} \in \mathbf{A}$. The set $\mathrm{A}$ is called convex cone if it satisfies that:

(1) $\lambda \boldsymbol{x} \in \mathrm{A} \quad$ if $\lambda \in \mathrm{R}, \lambda \geq 0$, and $\boldsymbol{x} \in \mathrm{A}$;

(2) $\boldsymbol{x}_{1}+\boldsymbol{x}_{2} \in \mathrm{A}$ if $\boldsymbol{x}_{1}$ and $\boldsymbol{x}_{2} \in \mathrm{A}$.

Let $B$ be a subset of $R^{n_{i}}$. $A$ set $B$ is said to span a convex cone $A$ if $B$ is a subset of $A$ and each vector of $A$ can be expressed as a finite linear combination of vectors of $B$ with non-negative coefficients. If convex cone $\mathrm{A}$ is spanned by a finite set, $\mathrm{A}$ is called polyhedral convex cone.

Let $\mathrm{A}$ be a finite subset of $\mathrm{R}^{n_{i}}$, and $\mathrm{A}=\left\{\boldsymbol{a}_{1}, \cdots, \boldsymbol{a}_{p}\right\}$. We denote by $\mathrm{A}^{L}$ the set consisting of all vectors $\boldsymbol{y} \in \mathrm{R}^{n_{i}}$ such that

$$
\boldsymbol{y}=\sum_{i=1}^{p} \lambda_{i} \boldsymbol{a}_{i} \text { and } \lambda_{i} \geq 0 \quad(i=1, \ldots, p)
$$

We can easily see that $A^{L}$ is a convex cone. We can also easily see that $A^{*}$ is a convex cone if $A$ is a convex cone, then $\mathrm{A}^{*}$ is called dual cone of a convex cone $\mathrm{A}$.

Let $\mathrm{A}$ be a convex cone. A is called vertex-convex cone if A does not contain subvector space of dimension $\mathrm{r}(1 \leq r \leq$ $\left.n_{i}\right)$.

As we mentioned in section $\mathbf{3 . 1}, f_{m i_{i}}(\boldsymbol{\theta})\left(i=1, \ldots, n_{i}\right)$ are linearly independent, hence the rank of the matrix

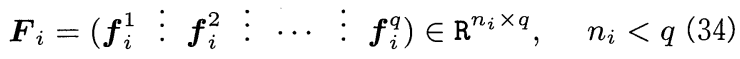

is $n_{i}$, which is the number of the terms of $\mathrm{i}$-th order principal minor. Then the subvector space spanned by $\left\{\boldsymbol{f}_{i}^{1}: \boldsymbol{f}_{i}^{2}: \ldots: \boldsymbol{f}_{i}^{q}\right\}$ is $n_{i}$-dimensional. Let $\Omega_{i}$ denote the set consisting of all indices of $\boldsymbol{f}_{i}^{j} \quad(1 \leq j \leq q)$ :

$$
\Omega=\{1,2, \cdots, q\}
$$

and $\varphi_{i}$ a subset of $\Omega_{i}$ that satisfies the followings:

(1) $\left\{\boldsymbol{f}_{i}^{j} \mid j \in \varphi_{i}\right\}$ spans a $\left(n_{i}-1\right)$-dimensional subspace that will be denoted by $\boldsymbol{W}_{\varphi_{i}}$;

(2) There is 1-dimensional vector $\boldsymbol{y}_{\varphi_{i}}$ in the orthogonal complement of $\boldsymbol{W}_{\varphi_{i}}\left(=\boldsymbol{W}_{\varphi_{i}}^{\perp}\right)$ such that $\boldsymbol{y}_{\varphi_{i}} \in$ $\left(\left\{\boldsymbol{f}_{i}^{1}, \quad \boldsymbol{f}_{i}^{2}, \cdots, \boldsymbol{f}_{i}^{q}\right\}^{L}\right)^{*}$ and non-zero.

Let $\mathcal{B}_{i}$ denote the family consisting of all the set $\varphi_{i} . \mathcal{B}_{i}$ is a finite set that has at most $2^{q}$ elements.

We now proceed to a important theorem:

Theorem 2: The set $D_{i}$ of the solutions of the system

$$
\boldsymbol{F}_{i}^{t} \boldsymbol{p}_{i} \geq \mathbf{o}
$$

is equal to the polyhedral vertex-convex cone

$$
\left\{\boldsymbol{y}_{\varphi_{i}} \mid \varphi_{i} \in \mathcal{B}_{i}\right\}^{L}
$$

Proof: We begin with the following lemma.

Lemma 2: $\quad \mathrm{D}_{i}=\left\{\boldsymbol{f}_{i}^{1}, \boldsymbol{f}_{i}^{2}, \cdots, \boldsymbol{f}_{i}^{q}\right\}^{*}$ is equal to the dual cone of $\left\{\boldsymbol{f}_{i}^{1}, \boldsymbol{f}_{i}^{2}, \cdots, \boldsymbol{f}_{i}^{q}\right\}^{\mathcal{L}}$, that is

$$
\mathrm{D}_{i}=\left(\left\{\boldsymbol{f}_{i}^{1}, \boldsymbol{f}_{i}^{2}, \cdots, \boldsymbol{f}_{i}^{q}\right\}^{L}\right)^{*} .
$$

Proof: $\quad$ Each $\boldsymbol{y} \in\left\{\boldsymbol{f}_{i}^{1}, \boldsymbol{f}_{i}^{2}, \cdots, \boldsymbol{f}_{i}^{q}\right\}^{<}$is described as $\boldsymbol{y}=\sum_{j=1}^{q} \boldsymbol{f}_{i}^{j} \lambda_{j}, \quad \lambda_{j} \geq 0$. Then, $\boldsymbol{y} \cdot \boldsymbol{x} \geq 0$ holds for each $\boldsymbol{x} \in \mathrm{D}_{i}$. Hence, if $\boldsymbol{x} \in \mathrm{D}_{i}$, then $\boldsymbol{x} \in\left(\left\{\boldsymbol{f}_{i}^{1}, \boldsymbol{f}_{i}^{2}, \cdots, \boldsymbol{f}_{i}^{q}\right\}^{L}\right)^{*}$, thereby $\mathrm{D}_{i} \subset\left(\left\{\boldsymbol{f}_{i}^{1}, \boldsymbol{f}_{i}^{2}, \cdots, \boldsymbol{f}_{i}^{q}\right\}^{L}\right)^{*}$.

Conversely, if $\quad \boldsymbol{x} \in\left(\left\{\boldsymbol{f}_{i}^{1}, \boldsymbol{f}_{i}^{2}, \cdots, \boldsymbol{f}_{i}^{q}\right\}^{L}\right)^{*}$, then $\boldsymbol{y} \cdot \boldsymbol{x} \geq 0 \quad$ holds for each $\boldsymbol{y} \in\left\{\boldsymbol{f}_{i}^{1}, \boldsymbol{f}_{i}^{2}, \cdots, \boldsymbol{f}_{i}^{q}\right\}^{L}$. Hence, $\quad \boldsymbol{f}_{i}^{j} \cdot \boldsymbol{x} \geq 0 \quad$ also holds for each $j=1, \cdots, q$. Therefore, if $\boldsymbol{x} \in\left(\left\{\boldsymbol{f}_{i}^{1}, \boldsymbol{f}_{i}^{2}, \cdots, \boldsymbol{f}_{i}^{q}\right\}^{L}\right)^{*}$, then $\boldsymbol{x} \in \mathrm{D}_{i}$, 
thereby, $\mathrm{D}_{i} \supset\left(\left\{\boldsymbol{f}_{i}^{1}, \boldsymbol{f}_{i}^{2}, \cdots, \boldsymbol{f}_{i}^{q}\right\}^{L}\right)^{*}$. Consequently, $\mathrm{D}_{i}=$ $\left(\left\{\boldsymbol{f}_{i}^{1}, \boldsymbol{f}_{i}^{2}, \cdots, \boldsymbol{f}_{i}^{q}\right\}^{L}\right)^{*}$.

Therefore, it is sufficient for us to obtain the dual cone of polyhedral convex cone $\left\{\boldsymbol{f}_{i}^{1}, \boldsymbol{f}_{i}^{2}, \cdots, \boldsymbol{f}_{i}^{q}\right\}^{<}$.

According to the following theorem:

Minkowsky-Farkas' Theorem [11]: The dual cone of polyhedral convex cone is polyhedral convex cone.

we can see that $\mathrm{D}_{i}$ is a polyhedral convex cone, and by Weyl's Theorem [11], it is shown that $\left\{\boldsymbol{y}_{\varphi_{i}} \mid \varphi_{i} \in \mathcal{B}_{i}\right\}$ span $\mathrm{D}_{i}$.

Finally, we will show that $\mathrm{D}_{i}$ is polyhedral vertex-convex cone. We begin with following lemma.

Lemma 3: The dual cone of $\left\{\boldsymbol{f}_{i}^{1}, \boldsymbol{f}_{i}^{2}, \cdots, \boldsymbol{f}_{i}^{q}\right\}$ is polyhedral vertex-convex cone if and only if the rank of the matrix

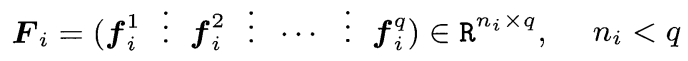

is equal to $n_{i}$.

Proof: We assume that the subspace spanned by $\boldsymbol{f}_{i}^{1}, \quad \boldsymbol{f}_{i}^{2}, \quad \cdots \boldsymbol{f}_{i}^{q} \in \mathrm{R}^{n_{i}}$ is r-dimensional and $r<n_{i}$, and denote that subspace by $U$ and the orthogonal complement of $\mathrm{U}$ by $\mathrm{U}^{\perp}$. Then each $\boldsymbol{y} \in \mathrm{R}^{n_{i}}$ can be described as $\boldsymbol{y}=\boldsymbol{u}+\boldsymbol{v}$ where $\boldsymbol{u} \in \mathrm{U}$ and $\boldsymbol{v} \in \mathrm{U}^{\perp}$. If $\boldsymbol{y}=\boldsymbol{u}+\boldsymbol{v}$ is a element of the dual cone of $\left\{\boldsymbol{f}_{i}^{1}, \boldsymbol{f}_{i}^{2}, \cdots \boldsymbol{f}_{i}^{q}\right\}$, then

$$
(\boldsymbol{u}+\boldsymbol{v}) \cdot \boldsymbol{f}_{i}^{j} \geq 0 \quad \text { for } \quad j=1, \cdots, q .
$$

That is

$$
\boldsymbol{u} \cdot \boldsymbol{f}_{i}^{j} \geq 0 \quad \text { for } \quad j=1, \cdots, q
$$

because

$$
v \in \mathrm{U}^{\perp}
$$

Hence, if $(\boldsymbol{u}+\boldsymbol{v}) \in\left(\left\{\boldsymbol{f}_{i}^{1}, \boldsymbol{f}_{i}^{2}, \cdots \boldsymbol{f}_{i}^{q}\right\}^{L}\right)^{*}$, then $\boldsymbol{u} \in$ $\left(\left\{\boldsymbol{f}_{i}^{1}, \boldsymbol{f}_{i}^{2}, \cdots \boldsymbol{f}_{i}^{q}\right\}^{L}\right)^{*}$. This implies

$$
\begin{aligned}
& \left.\left(\begin{array}{lll}
\left\{\boldsymbol{f}_{i}^{1},\right. & \boldsymbol{f}_{i}^{2}, & \cdots \boldsymbol{f}_{i}^{q}
\end{array}\right\}^{\perp}\right)^{*} \\
= & \left.\left(\begin{array}{lll}
\left\{\boldsymbol{f}_{i}^{1},\right. & \boldsymbol{f}_{i}^{2}, & \cdots \boldsymbol{f}_{i}^{q}
\end{array}\right\}^{\perp}\right)^{*} \cap \mathrm{U}+\mathrm{U}^{\perp} .
\end{aligned}
$$

If the dual cone $\left(\left\{\boldsymbol{f}_{i}^{1}, \boldsymbol{f}_{i}^{2}, \cdots \boldsymbol{f}_{i}^{q}\right\}^{L}\right)^{*}$ has a subspace of dimension 1 , then the dual cone has 2 elements $\boldsymbol{b}$ and $-\boldsymbol{b}$ among the elements spanning the dual cone, where $\boldsymbol{b}$ is a basis of the 1-dimensional subspace. Then $\boldsymbol{b}$ satisfies

$$
\boldsymbol{b} \cdot \boldsymbol{f}_{i}^{j} \geq 0 \text { and }-\boldsymbol{b} \cdot \boldsymbol{f}_{i}^{j} \geq 0 \text { for } j=1, \cdots, q .
$$

Hence, $\boldsymbol{b} \cdot \boldsymbol{f}_{i}^{j}=0$ holds for $j=1, \cdots, q$. This implies that the 1 -dimensional subspace is involved in $\mathrm{U}^{\perp}$. If the dual cone has a subspace of dimension $t$ and $1<t<r$, the subspace will be shown to be involved in $\mathrm{U}^{\perp}$ in a similar way.
We defined that polyhedral vertex-convex cone does not contain a subspace of dimension $\mathrm{t}(t \geq 1)$. Then the dual cone is polyhedral vertex-convex cone if and only if $\mathrm{U}^{\perp}$ is empty, and $\mathrm{U}^{\perp}$ is empty if and only if the rank of $\boldsymbol{F}_{i}$ is $n_{i}$.

The rank of $\boldsymbol{F}_{i}$ is $n_{i}$ as we mentioned above, hence by Lemma 3 the dual cone is polyhedral vertex-convex cone.

Though we can obtain the set of the solutions of the system $\boldsymbol{F}_{i}^{t} \boldsymbol{p}_{i} \geq \mathbf{o}$ by finite calculations from Theorem 2 , we must examine the set because what we should obtain is the set of the solutions of the system $\boldsymbol{F}_{i}^{t} \boldsymbol{p}_{i}>\mathbf{o}$, not $\boldsymbol{F}_{i}^{t} \boldsymbol{p}_{i} \geq \mathbf{o}$ We will examine the set below.

The dual cone of $\left\{\boldsymbol{f}_{i}^{1}, \boldsymbol{f}_{i}^{2}, \cdots \boldsymbol{f}_{i}^{q}\right\}$ is the intersection of finitely many halfspaces

$$
\bigcap_{j=1}^{q}\left\{\boldsymbol{x} \in \mathrm{R}^{n_{i}} \mid \boldsymbol{f}_{i}^{j} \cdot \boldsymbol{x} \geq 0\right\}
$$

whose boundary hyperplanes are

$$
\left\{\boldsymbol{x} \in \mathrm{R}^{n_{i}} \mid \boldsymbol{f}_{i}^{j} \cdot \boldsymbol{x}=0\right\} \quad(1 \leq j \leq q) .
$$

Then let $\mathbf{Q}_{i}^{j}$ denote the subset of $\mathrm{R}^{n_{i}}$ such that

$$
\left\{\boldsymbol{x} \in \mathrm{R}^{n_{i}} \mid \boldsymbol{f}_{i}^{j} \cdot \boldsymbol{x}>0\right\},
$$

and $\mathrm{S}_{i}^{j}$ the hyperplane of $\mathrm{R}^{n_{i}}$ such that

$$
\left\{\boldsymbol{x} \in \mathrm{R}^{n_{i}} \mid \boldsymbol{f}_{i}^{j} \cdot \boldsymbol{x}=0\right\} .
$$

Because of the reason just mentioned above, we can describe the dual cone as

$$
\mathrm{D}_{i}=\bigcap_{j=1}^{q}\left(\mathrm{Q}_{i}^{j} \bigcup \mathrm{S}_{i}^{j}\right)
$$

hence, by short calculation, we can obtain

$$
\mathrm{D}_{i}=\left\{\bigcap_{j=1}^{q} \mathrm{Q}_{i}^{j}\right\} \bigcup_{k=2}^{q}\left[\left\{\bigcap_{s=1}^{k-1} \mathrm{Q}_{i}^{s}\right\} \bigcap\left\{\mathrm{S}_{i}^{k} \bigcap \mathrm{D}_{i}\right\}\right] \bigcup\left\{\mathrm{S}_{i}^{1} \bigcap \mathrm{D}_{i}\right\}
$$

Therefore, we can see that the dual cone $\mathrm{D}_{i}$ is constituted of the interior $\left\{\bigcap_{j=1}^{q} \mathrm{Q}_{i}^{j}\right\}$, which is the subset of the solutions of the system $\boldsymbol{F}_{i}^{t} \boldsymbol{p}_{i}>\mathbf{o}$, and boundary that is union of such subset of hyperplanes that defined in (47). We can easily see that the interior is convex set.

From the examination above, we also see that the hyperplanes whose subset constitute the boundary play very important role when we make use of the convex cone. Hence, it would be worth while to obtain such hyperplanes.

We use the following theorem: 
Farkas' Theorem [11]: If A is a finite set of vectors, then $\mathrm{A}^{* *}=\mathrm{A}^{L}$.

Applying Farkas' Theorem to $\left\{\boldsymbol{f}_{i}^{1}, \boldsymbol{f}_{i}^{2}, \cdots \boldsymbol{f}_{i}^{q}\right\}$, which is a finite set, and taking Lemma 2 into account, we obtain

$$
\left.\left\{\boldsymbol{f}_{i}^{1}, \quad \boldsymbol{f}_{i}^{2}, \cdots \boldsymbol{f}_{i}^{q}\right\}^{L}=\left(\begin{array}{llll}
\left\{\boldsymbol{f}_{i}^{1},\right. & \boldsymbol{f}_{i}^{2}, & \cdots & \boldsymbol{f}_{i}^{q}
\end{array}\right\}^{\llcorner}\right)^{* *} .
$$

By Theorem 2, the dual cone of $\left\{\boldsymbol{f}_{i}^{1}, \boldsymbol{f}_{i}^{2}, \cdots \boldsymbol{f}_{i}^{q}\right\}^{\llcorner}$is polyhedral vertex-convex cone that is described as $\left\{\boldsymbol{y}_{\varphi_{i}} \mid \varphi_{i} \in\right.$ $\left.\mathcal{B}_{i}\right\}^{<}$. We will apply Theorem 2 again. For the purpose, we number the elements of $\left\{\boldsymbol{y}_{\varphi_{i}} \mid \varphi_{i} \in \mathcal{B}_{i}\right\}^{\swarrow}$, and express them as $\left\{\boldsymbol{y}_{i}^{1}, \boldsymbol{y}_{i}^{2}, \cdots, \boldsymbol{y}_{i}^{s}\right\} \quad\left(s \leq 2^{q}\right)$. Let $\Omega \boldsymbol{y}_{i}$ denote the set of all indices of $\boldsymbol{y}_{i}^{j}$, then $\Omega \boldsymbol{y}_{i}=\{1,2, \cdots s\}$, and $\xi_{i}$ a subset of $\Omega \boldsymbol{y}_{i}$ that satisfies the followings:

(1) $\left\{\boldsymbol{y}_{i}^{j} \mid j \in \xi_{i}\right\}$ spans a $\left(n_{i}-1\right)$-dimensional subspace that will be denoted by $\boldsymbol{W}_{\xi_{i}}$;

(2) There is 1-dimensional vector $\boldsymbol{v}_{\xi_{i}}$ in the orthogonal complement of $\boldsymbol{W}_{\xi_{i}}\left(=\boldsymbol{W}_{\xi_{i}}^{\perp}\right)$ such that $\boldsymbol{v}_{\xi_{i}} \in$ $\left(\left\{\boldsymbol{y}_{i}^{1}, \boldsymbol{y}_{i}^{2}, \cdots, \boldsymbol{y}_{i}^{s}\right\}^{\llcorner}\right)^{*}$ and non-zero.

Let $\mathcal{C}_{i}$ denote the family consisting of all the set $\xi_{i}$. From Theorem 2, we obtain

$$
\left\{\boldsymbol{f}_{i}^{1}, \quad \boldsymbol{f}_{i}^{2}, \cdots \boldsymbol{f}_{i}^{q}\right\}^{\mathcal{L}}=\left\{\boldsymbol{v}_{\xi_{i}} \mid \xi_{i} \in \mathcal{C}_{i}\right\}^{<},
$$

hence, we can conclude that each element spanning the convex cone $\left\{\boldsymbol{f}_{i}^{1}, \boldsymbol{f}_{i}^{2}, \quad \cdots \boldsymbol{f}_{i}^{q}\right\}^{<}$corresponds to one of the elements of $\left\{\boldsymbol{v}_{\xi_{i}} \mid \xi_{i} \in \mathcal{C}_{i}\right\}$ and they would coincide if they were normalized. The hyperplanes whose subsets constitute the boundary are the set $\left\{\boldsymbol{W}_{\xi_{i}} \mid \xi_{i} \in \mathcal{C}_{i}\right\}$ because each of them is orthogonal complement of the element which spans the convex cone $\left\{\boldsymbol{f}_{i}^{1}, \boldsymbol{f}_{i}^{2}, \cdots \boldsymbol{f}_{i}^{q}\right\}^{L}$.

We obtained $\mathrm{N}$ polyhedral vertex-convex cones and the vectors $\boldsymbol{p}_{i}=\left[\begin{array}{llll}p_{m i_{1}} & p_{m i_{2}} & \cdots & p_{m i_{i}}\end{array}\right]^{t}$ for $i=$ $1,2, \cdots, N$, each of the vectors corresponds to the cone. Each polyhedral vertex-convex cone can be obtained by finite calculations. Here, we state the main result of this section:

A set of base-parameter values is "possible" if and only if each $\boldsymbol{p}_{i}$ that is determined by the set of base-parameter values exists in the domain that is determined from $\boldsymbol{f}_{i}$, and the domain is approximately the interior of the polyhedral vertex-convex cone corresponding to $\boldsymbol{F}_{i}$.

\section{Modification of Estimated Base-Parameter Values}

After estimating a set of base-parameter values, we will be able to determine $\boldsymbol{p}_{i}$ for $i=1,2, \cdots, N$ as the constant vectors whose entries are real. Each constant vector $\boldsymbol{p}_{i}$ must be a element of the subset that is determined by $\boldsymbol{f}_{i}$, otherwise the inertial matrix would not always be positive definite, that would be physically impossible. Nevertheless, we may estimate such a set of base-parameter values that determine $\boldsymbol{p}_{i}$ which is outside of the cone. If we use such a set of base-parameter values, we can not ensure good control performance of the manipulator. Hence, after estimating a set of base-parameter values we should judge if it is "possible" or not, and modify the estimated base-parameter values for the set of them to be at least "possible" if it is judged not to be. Then in this chapter we propose one method to do it using the convex cone on computers.

\subsection{Judgement}

Let $\hat{\boldsymbol{p}}_{i}$ for $i=1,2, \cdots, N$ denote the constant vector whose entries are determined by a set of estimated base-parameter values. A simple method to judge if it is "possible" or not is to examine each $\hat{\boldsymbol{p}}_{i}$ if

$$
\boldsymbol{v}_{\xi_{i}} \cdot \hat{\boldsymbol{p}}_{i}>0
$$

holds for each $\xi_{i} \in \mathcal{C}_{i}$ and for $i=1,2, \cdots, N$.

\subsection{A Method to Modify}

If the set of estimated base-parameter values is judged not to be "possible", there is at least one $\hat{\boldsymbol{p}}_{i}$ among $\hat{\boldsymbol{p}_{1}}, \hat{\boldsymbol{p}_{2}}, \cdots, \hat{\boldsymbol{p}_{N}}$ that does not hold $\boldsymbol{v}_{\xi_{i}} \cdot \hat{\boldsymbol{p}_{i}}>0$ for each $\xi_{i} \in \mathcal{C}_{i}$. Then let $\Lambda$ denote the set consisting of all indices: $1,2, \cdots, N$, and $\Gamma$ the subset of $\Lambda$ which satisfy that for $j \in \Gamma, \boldsymbol{v}_{\xi_{j}} \cdot \hat{\boldsymbol{p}}_{j} \leq 0$ holds for some $\xi_{j} \in \mathcal{C}_{j}$. Let $\mathcal{E}_{j}$ denote the subset of $\mathcal{C}_{j},(j \in \Gamma)$ which satisfy that for $\xi_{j} \in \mathcal{E}_{j}$, $\boldsymbol{v}_{\xi_{j}} \cdot \hat{\boldsymbol{p}}_{j} \leq 0$ holds.

We propose a method to modify the estimated baseparameter values taking the following steps.

Step1: We find the nearest point on the boundary of convex cone corresponding to $\boldsymbol{p}_{i}$ for $i \in \Gamma$ from the point $\hat{\boldsymbol{p}}_{\boldsymbol{i}}$. We use "nearest" in the sense of Euclidean distance. The nearest point would be easily found because we obtained hyperplanes $\boldsymbol{W}_{\xi_{i}} \quad\left(\xi_{i} \in \mathcal{C}_{j}\right)$ whose subset constitute the boundary of convex cone, and it would be sufficient to examine the only hyperplanes $\boldsymbol{W}_{\xi_{i}}$ for $\xi_{i} \in \mathcal{E}_{i}$ because $\hat{\boldsymbol{p}}_{i}$ is near them outside the convex cone.

Let $\tilde{\boldsymbol{p}}_{i}(i \in \Gamma)$ denote the nearest point on the boundary of convex cone.

Step2: We obtain a line through the point $\hat{\boldsymbol{p}}_{i}$ and $\tilde{\boldsymbol{p}}_{i}$ for $i \in \Gamma$, and take another point $\breve{\boldsymbol{p}_{i}}$ in the interior of the convex cone and on the line.

Step3: We make a hypercube in the interior, taking its center on $\breve{\boldsymbol{p}}_{i}$ and the length of edges as $2 \alpha_{i}$ for each $i \in \Gamma$. In the same way we make a hypercube, taking its center on $\hat{\boldsymbol{p}}_{i}$ for each $i \notin \Gamma$. Thereby we obtain a inequality for each element of $\boldsymbol{p}_{i}$ for each $i \in \Lambda$. It is described as

$$
\breve{p}_{m i v_{i}}-\alpha_{i} \leq p_{m i v_{i}} \leq \breve{p}_{m i v_{i}}+\alpha_{i}
$$


for each $i \in \Gamma$,

$$
\tilde{p}_{m i v_{i}}-\alpha_{i} \leq p_{m i v_{i}} \leq \tilde{p}_{m i v_{i}}+\alpha_{i}
$$

for each $i \notin \Gamma$, for $1 \leq v_{i} \leq n_{i}$.

Step4: We search the system of inequalities in Step3 for a set of base-parameter values. After searching, if we fail to find the solution, we take $\alpha_{i}$ as $\alpha_{i}+\varepsilon_{i}, \varepsilon_{i}$ is appropriate positive value, and go back to Step3.

If we make the domain in each convex cone, for which a solution is searched, enlarge so as to gradually cover the interior of the convex cone, we will surely be able to obtain a solution. Because each $\dot{p}_{i}$ that is determined by the set of true base-parameter values is in the interior for each $1 \leq i \leq N$, and $p_{m i_{i}}$ are continuous on the continuous changes of the base-parameter values as mentioned in chapter 3 , there is a domain in each convex cone that is the neighborhood of $\boldsymbol{p}_{i}$.

It is needed to find a good algorithm for Step4 to search for a solution, but it should be done after the structure of the manipulator is fixed because the algorithm needs the information about the explicit forms of $p_{m i_{i}}$.

\section{Conclusion}

We proposed a method to judge if a set of base-parameter values determines the inertial matrix to be positive definite for each configuration of the manipulator or not, when we approximately consider the continuous change of each joint variable of the manipulator as a finite set of discrete points. The method can be executed on computers. Using this method we can judge if a set of estimated base-parameter values is "possible" or not. If a set of base-parameter values is not "possible", it is physically impossible. Also we proposed one method to modify the estimated base-parameter values for the set of them to be at least "possible" if we judge it is not.
Though we used approximation in obtaining the subset of the solutions of each inequality in (12), we could make the accuracy of approximation high enough for the purpose of judgement and modification of the set of estimated baseparameter values. Obtaining a good algorithm in Step4 in chapter 4 would be very important.

\section{References}

[1] C.H. An, C.G. Atkeson and J.M. Hollerbach: Model-Based Control of a Robot Manipulator, The MIT Press, 1988.

[2] H. Mayeda, K. Yoshida and K. Osuka: "Base parameters of Manipulator Dynamic Model," IEEE J. of Robotics and Automation, vol. 6 , no. 3, pp. 312-321, 1990.

[ 3 ] H. Mayeda and K. Ohashi: "Base parameters of Dynamic Models for General Open Loop Kinematic Chains," Robotics Research 5, pp. 271-278, 1990.

[4] H. Mayeda, K. Osuka and A. Kangawa: "A New Identification Method for Serial Manipulator Arms," Proc. 9th IFAC World Congress, pp. 2429-2434, 1984.

[ 5 ] H. Kawasaki and K. Nishimura: "Parameter Identification of Mechanical Manipulators," SICE Trans., vol. 22, no. 1, pp. 76-83, 1986. (in Japanese)

[6] P.K. Khosla: "Real-Time Control and Identification of DirectDrive Manipulators," Ph. D. dissertation, Carnegie-Mellon Univ., Pittsburgh, PA, Aug. 1986.

[7] C.G. Atkeson, C.H. An and J.M. Hollerbach: "Estimation of Inertial Parameters of Manipulator Loads and Links," Int. J. Robotics Research, vol. 5, no. 3, pp. 101-119, 1986.

[8] H. Mayeda, M. Maruyama, K. Yoshida, N. Ikeda and O. Kuwaki: "Experimental Examination of the Identification Methods for an Industrial Robot Manipulator," Experimental Robotics II: Lecture Notes in Control and Information Sciences 190, R. Chatila and G. Hirzinger, ed., pp. 546-560, Springer-Verlag, 1993.

[9] K. Yoshida, N. Ikeda and H. Mayeda: "Experimental Study of the Identification Methods for an Industrial Robot Manipulator," Proc. of Int. Conf. of IROS'92, pp. 263-270, 1992.

[10] M. Renaud: "Quasi-Minimal Computation of the Dynamic Model of Robot Manipulator Utilising the Newton-Euler Formalize and the Notion of Augmented Body," Proc. IEEE Int. Conf. Robotics and Automation, pp. 1677-1682, 1987.

[11] H.W. Kuhn and A.W. Tucker (Eds.): Linear Inequalities and Related Systems, Annals of Math. Studies No. 38, Princeton Unversity Press, 1956.

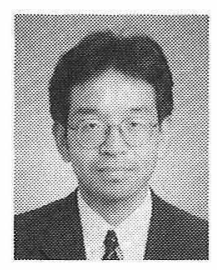

\section{Koji Yoshida}

was born on May 5, 1961. He received the M.S. degree in control engineering from Osaka University in 1988. He joined Toshiba Corporation in 1988, and in 1993, he joined Osaka Prefecture University, where he is currently a Research Associate of the Department of Mechanical Systems Engineering. His current research interests are in robotics. He is a Member of the Robotics Society of Japan, the Japan Society of Mechanical Engineering, and the IEEE.

(日本ロボット学会正会員)

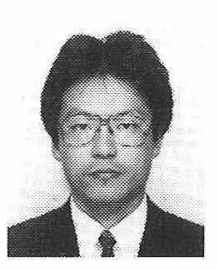

\section{Koichi Osuka}

was born on Nov. 16, 1959. He received the M.S. degree in control engineering from Osaka University in 1984. He joined the R\&D Center, Toshiba Corporation, in 1984, and in 1986, he joined the University of Osaka Prefecture, where he is now an Associate Professor of the Department of Mechanical Systems Engineering. His current research interests are in robotics and robust control of mechanical systems. Dr. Osuka is a member of the Robotics Society of Japan, the Society of Instrument and control Engineering, the Institute of System, Control and Information Engineering, and the Japan Society of Mechanical Engineering. （日本ロボット学会正会員） 


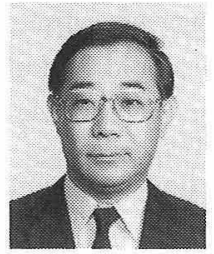

\section{Hirokazu Mayeda}

was born in Osaka, Japan on Oct.5, 1943. He received the B.S., M.S., and doctral degrees in control engineering from Osaka University in 1966, 1968, and 1972, respectively. Since1971, he was an Assistant Professor and an Associate Professor of the Department of Control Engineering, Osaka University, and currently he is a Professor of the Department of Computer \& Systems Engineering, Ritsumeikan University. From 1978 to 1979, he was a Visiting Scientist in the Department of Electrical Engineering, the University of Toront, Canada. His professional interests are in robotics and robot control, and currently in the task understanding of robot tasks and the open controller for robots. Dr. Mayeda is a member of the Robotics Society of Japan, the Society of Instrument and Control Engineering, the Institute of System, Control and Information Engineering, the Japan Society of Mechanical Engineering, and the IEEE.

(日本ロボット学会正会員)

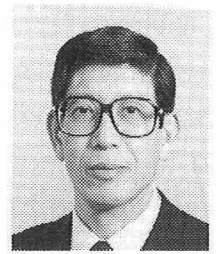

\section{Toshiro Ono}

was born on Nov. 22, 1936. He received the B.S. degree in mechanical engineering from Osaka Prefecture University in 1960, the M.S. and $\mathrm{Ph} . \mathrm{D}$ degrees in aeronautical engineering from Kyoto University in 1962, and 1966, respectively. He joined Kyoto University in 1965 as a Research Associate, and in 1967 he was appointed as a Lecturer of the Department of Applied Mathematics and Physics. In 1968, he joined Osaka Prefecture University, where he is currently a Professor of the Department of Mechanical Systems Engineering. His current research interests are directed to application-oriented problems of stochastic control theory, estimation theory, dynamic mass measurement problems, and robotics for welfare engineering. Dr. Ono is a member of the Robotics Society of Japan, the Society of Instrument and Control Engineers, the Institute of Systems, Control and Information Engineers, and the Japan Society of Mechanical Engineers, etc..

（日本ロボット学会正会員） 